Moroccan J. of Pure and Appl. Anal. (MJPAA)

Volume 7(3), 2021, Pages 448-460

ISSN: Online 2351-8227 - Print 2605-6364

DOI: $10.2478 / \mathrm{mjpaa}-2021-0030$

\title{
On the generalized fractional Laplace transform
}

\author{
VIRENDRA KUMAR ${ }^{1}$
}

АвsтRACт. In the present paper a generalization of the Laplace transform is introduced and studied. Its inversion formula is also obtained. As an application, we obtain the generalized fractional Laplace transform of a general class of functions and a product of the Fox's $H$ - function and general class of functions. The results obtained are of general nature and capable of yielding a large number of known or new results as special cases. For illustration, some special cases involving important special functions are mentioned.

Mathematics Subject Classification (2020). 44A10, 44A20.

Key words and phrases. Laplace transform, $H$-function, Struve's function, Lommel's function, MittagLeffler functioin, Riemann-zeta function.

\section{Introduction}

Several scholars including Bhonsle [1, 2], Gupta and Agrawal [7], Goyal and Vasishta [8], Goyal and Jain [9], Kumar [16, 17, 18], Srivastava [28, 29, 30], Srivastava and Tuan [32], Srivastava and Yürekli [33] and Yakubovich and Martins [35] have studied and explored Laplace, Meijer, Stieltjes, Hankel and $H$-function transforms at large in the form of generalizations, convolution and connecting theorems. F. Jarad and T. Abdeljawad [12] have introduced, studied and explored the generalized Laplace transform and applied the same for the generalized fractional integrals and derivatives, and to solve some generalized fractional differential equations in the frame of derivatives of a function with respect to another function. In this paper we study the generalized fractional Laplace transform. Since the $H$-function and general class of

Received : October 01, 2020 - Accepted: April 08, 2021.

(C) The Author(s) 2021. This article is published with open access by Sidi Mohamed Ben Abdallah University.

${ }^{1}$ Formerly Scientist-B, Defence Research and Development Organization, New Delhi, India.

Address for correspondence: D-436, Shastri Nagar, Ghaziabad-201002, India.

e-mail: vkumar10147@yahoo.com. 
functions are reducible to very important special functions being widely used in Mathematics, Engineering and Mathematical Physics, the generalized fractional Laplace transform of some special functions is obtained as special cases of the main results.

Now, we mention important definitions.

Definition 1.1. The Laplace transform [6] of a function $f(x) \in(0, \infty)$ is defined by the following equation:

$$
F(c)=L\{f(x) ; c\}=\int_{0}^{\infty} e^{-c x} f(x) d x,
$$

where $c$ is a complex variable. $f(x)$ is called the inverse Laplace transform of $F(c)$ and is written as follows:

$$
f(x)=L^{-1}\{F(c)\}
$$

Definition 1.2. If $f(x)$ has a continuous derivative and is of exponential order and if $F(c)=L\{f(x)\}$, then the inversion formula [6] for the classical Laplace transform defined by (1.1) is given by the following equation:

$$
f(x)=\frac{1}{2 \pi i} \int_{\gamma-i \infty}^{\gamma+i \infty} e^{c x} F(c) d c, x>0
$$

and

$$
f(x)=0, x<0 .
$$

Definition 1.3. The generalized fractional Laplace transform of a function $f(x) \in(0, \infty)$ is hereby introduced and defined by the following equation:

$$
\phi(c)=L^{*}\{f(x) ; c\}=\int_{0}^{\infty} e^{-c^{\mu / \rho} x} f(x) d x,
$$

where $c$ is a complex variable. $\mu$ and $\rho$ are positive real numbers.

Remark 1.1. The integral transform defined by (1.5) is a new generalization of (1.1). If we substitute $\mu=1$, the equation (1.5) reduces to the following fractional Laplace transform [24, 27]:

$$
\tilde{f}_{\rho}(c)=L_{\rho}\{f(x) ; c\}=\int_{0}^{\infty} e^{-c^{1 / \rho} x} f(x) d x,
$$

where $c$ is a complex variable and $\rho$ is positive real number. If we substitute $\mu=1$ and $\rho=1$, the equation (1.5) reduces to (1.1).

Remark 1.2. The relation between the generalized fractional Laplace transform and classical Laplace transform is as follows:

$$
L^{*}\{f(x) ; c\}=L\{f(x) ; s\}, s=c^{\mu / \rho} .
$$

Definition 1.4. Fox's H-function occurring in this paper is defined and represented by means of the following Mellin-Barnes type contour integral [31]:

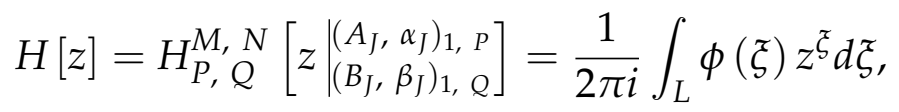


where $i=\sqrt{-1}, L$ is a contour which goes from $\gamma-i \infty$ to $\gamma+i \infty, z \neq 0$. and

$$
(\phi \xi)=\frac{\prod_{J=1}^{M} \Gamma\left(B_{J}-\beta_{J} \xi\right) \prod_{J=1}^{N} \Gamma\left(1-A_{J}+\alpha_{J} \xi\right)}{\prod_{J=M+1}^{Q} \Gamma\left(1-B_{J}+\beta_{J} \xi\right) \prod_{J=N+1}^{P} \Gamma\left(A_{J}-\alpha_{J} \xi\right)} .
$$

For the convergence, existence conditions and other details of the above Fox's H-function, one may refer to the books written by Srivastava, Gupta and Goyal [31]; Kilbas and Saigo [21]; Mathai, Saxena and Haubold [23] and Prudnikov, Brychkov and Marichev [25].

Definition 1.5. The author introduced a general class of functions defined in the following manner [13] (see also $[14,15,19,20])$ :

$$
\begin{aligned}
V_{n}(x) & =V_{n}^{h_{m}, d, g_{j}}\left[p, \tau, k, w, q, k_{m}, a_{j}, b_{r}, \alpha, \beta, \delta ; x\right] \\
& =\lambda \sum_{n=0}^{\infty} \frac{(-p)^{n} \prod_{m=1}^{t}\left[\left(h_{m}\right)_{n+k_{m}}\right](d+\alpha n+\beta)^{-\tau}(x / 2)^{n k+d w+q}}{\prod_{j=1}^{s}\left[\left(g_{j}\right)_{n+a_{j}}\right] \prod_{r=1}^{u}\left[(d)_{\alpha n \delta+b_{r}}\right]},
\end{aligned}
$$

where

(i) $p, k, w, q, \beta, \delta, k_{m}, a_{j}, b_{r}(m=1, \ldots, t ; j=1, \ldots, s ; r=1, \ldots, u)$ are real numbers.

(ii) $t, s$ and $u$ are natural numbers.

(iii) $h_{m}, g_{j} \geq 1(m=1, \ldots, t ; j=1, \ldots, s)$ and $d$ may be real or complex.

(iv) $\alpha>0, \operatorname{Re}(\tau)>0, \operatorname{Re}(d)>0, x$ is a variable and $\lambda$ is an arbitrary constant.

(v) The series on the right hand side of (1.10) converges absolutely if $t<s$ or $t=s$ with $\left|p(x / 2)^{k}\right| \leq 1$.

For details of convergence conditions of the series on the right hand side of (1.10) one may refer to the paper [14].

Remark 1.3. The general class of functions defined by (1.10) is quite general in nature as it unifies and extends a number of useful functions such as unified Riemann-zeta function [10], generalized hypergeometric function [3], Bessel function [4], Wright's generalized Bessel function [35], Struve's function [4], Lommel's function [4], generalized Mittag-Leffler function [26], exponential function, sine function, cosine function and MacRobert's E-function [3] etc. (see, e.g. [13, 15]).

\section{Inversion formula}

In this section we obtain the inversion formula for the generalized fractional Laplace transform defined by (1.5).

Theorem 2.1. If $f(x)$ has a continuous derivative and is of exponential order and if $\phi(c)=L^{*}\{f(x)\}$, then the inversion formula for the generalized fractional Laplace transform is defined by

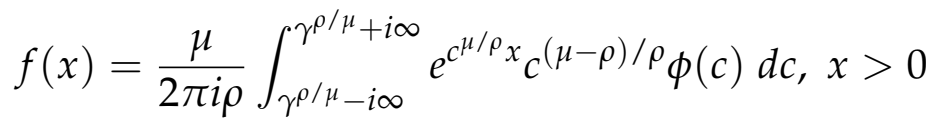


and

$$
f(x)=0, x<0,
$$

where $\mu$ and $\rho$ are positive real numbers and $\rho \leq \mu$.

Proof. Let $\phi(x)$ be absolutely integrable in $(-\infty, \infty)$, then from Fourier integral formula we have

$$
\phi(x)=\frac{1}{2 \pi} \int_{-\infty}^{\infty} e^{i \omega x}\left\{\int_{-\infty}^{\infty} \phi(z) e^{-i \omega z} d z\right\} d \omega
$$

Now, let us take

$$
\phi(x)=\left\{\begin{array}{c}
e^{-\gamma x} f(x), x>0 \\
0, x<0
\end{array}\right.
$$

For $x>0$, we get

$$
e^{-\gamma x} f(x)=\frac{1}{2 \pi} \int_{-\infty}^{\infty} e^{i \omega x}\left\{\int_{-\infty}^{\infty} e^{-(\gamma+i \omega) z} f(z) d z\right\} d \omega, \quad \gamma>x
$$

Now, we substitute $\gamma+i \omega=c^{\mu / \rho}$ and we get

$$
e^{-\gamma x} f(x)=\frac{\mu}{2 \pi i \rho} \int_{\gamma^{\rho / \mu}-i \infty}^{\gamma^{\rho / \mu}+i \infty} e^{\left(c^{\mu / \rho}-\gamma\right) x}\left\{\int_{-\infty}^{\infty} e^{-c^{\mu / \rho} z} f(z) d z\right\} c^{(\mu-\rho) / \rho} d c
$$

Now, using equation (1.5), we get

$$
e^{-\gamma x} f(x)=\frac{\mu}{2 \pi i \rho} \int_{\gamma^{\rho / \mu}-i \infty}^{\gamma^{\rho / \mu}+i \infty} e^{\left(c^{\mu / \rho}-\gamma\right) x_{c}(\mu-\rho) / \rho} \phi(c) d c
$$

After a little simplification we arrive at the desired result (2.1).

Remark 2.1. Obviously when $\mu=1,(2.1)$ reduces to the inversion formula for alfa-integral Laplace transform studied by Medina et al. [24] which further reduces to the inversion formula (1.3) for conventional Laplace transform (1.1) when $\rho=1$.

\section{Main theorems}

In this section we obtain the images of the general class of functions and a product of the Fox's $H$-function and general class of functions in the generalized fractional Laplace transform defined by (1.5). 
Theorem 3.1. If $\mu, \rho, \eta$ are positive real numbers, $\operatorname{Re}(c)>0$ and $\operatorname{Re}\{d+\eta(n k+d w+q)\}>-1$, then the following result holds:

$$
\begin{aligned}
& L^{*}\left\{x^{d} V_{n}^{h_{m}, d, g_{j}}\left[p, \tau, k, w, q, k_{m}, a_{j}, b_{r}, \alpha, \beta, \delta ; y x^{\eta}\right] ; c\right\} \\
& =\int_{0}^{\infty} e^{-c^{\mu / \rho} \rho_{x}} x^{d} V_{n}^{h_{m}, d, g_{j}}\left[p, \tau, k, w, q, k_{m}, a_{j}, b_{r}, \alpha, \beta, \delta ; y x^{\eta}\right] d x \\
& =\lambda \sum_{n=0}^{\infty} \frac{(-p)^{n} \prod_{m=1}^{t}\left[\left(h_{m}\right)_{n+k_{m}}\right](d+\alpha n+\beta)^{-\tau}(y / 2)^{n k+d w+q} \Gamma\{d+\eta(n k+d w+q)+1\}}{\prod_{j=1}^{s}\left[\left(g_{j}\right)_{n+a_{j}}\right] \prod_{r=1}^{u}\left[(d)_{\alpha n \delta+b_{r}}\right] c^{\mu\{d+\eta(n k+d w+q)+1\} / \rho}},
\end{aligned}
$$

provided that the conditions mentioned with (1.10) are satisfied.

Proof. We first express the general class of functions occurring in the left hand side of (3.1) in series form with the help of (1.10) and then interchange the order of integration and summation which is permissible since the series occurring in (1.10) is absolutely convergent. Now, we put $c^{\mu / \rho} x=\xi$ and we get left hand side (say $\Delta$ ) as follows:

$$
\begin{aligned}
\Delta & =\lambda \sum_{n=0}^{\infty} \frac{(-p)^{n} \prod_{m=1}^{t}\left[\left(h_{m}\right)_{n+k_{m}}\right](d+\alpha n+\beta)^{-\tau}(y / 2)^{n k+d w+q}}{\prod_{j=1}^{s}\left[\left(g_{j}\right)_{n+a_{j}}\right] \prod_{r=1}^{u}\left[(d)_{\alpha n \delta+b_{r}}\right]} \\
& \times \frac{1}{c^{\mu\{d+\eta(n k+d w+q)+1\} / \rho} \int_{0}^{\infty} e^{-\xi} \xi^{d+\eta(n k+d w+q)} d \xi,}
\end{aligned}
$$

where $\mu, \rho, \eta$ are positive real numbers, $\operatorname{Re}(c)>0, \operatorname{Re}\{d+\eta(n k+d w+q)\}>-1$ and the conditions mentioned with (1.10) are satisfied.

Now, using the following result [3, p. 1, Eq. (1)] in (3.2)

$$
\int_{0}^{\infty} e^{-x} x^{\lambda-1} d x=\Gamma(\lambda), \quad \operatorname{Re}(\lambda)>0
$$

we arrive at the desired result (3.1).

Theorem 3.2. If

(i) $\mu, \rho, \eta, \sigma$ are positive real numbers,

(ii) $\operatorname{Re}(c)>0, A>0,|\arg z|<\frac{1}{2} A \pi, \delta>0$ and 
(iii) $\operatorname{Re}\left\{\zeta+\eta(n k+d w+q)+\sigma \min _{1 \leq J \leq M}\left(\frac{B_{J}}{\beta_{J}}\right)\right\}>-1$, then the following result holds

$$
\begin{aligned}
& L^{*}\left\{x^{\zeta} H_{P, Q}^{M, N}\left[z x^{\sigma}\left[\begin{array}{l}
\left(A_{J}, \alpha_{J}\right)_{1, P} \\
\left(B_{J}, \beta_{J}\right)_{1, Q}
\end{array}\right] V_{n}^{h_{m}, d_{,} g_{j}}\left[p, \tau, k, w, q, k_{m}, a_{j}, b_{r}, \alpha, \beta, \delta ; y x^{\eta}\right]\right\}\right. \\
& =\int_{0}^{\infty} e^{-c^{\mu / \rho} x} x^{\zeta} H_{P, Q}^{M, N}\left[z x^{\sigma}\left[\begin{array}{l}
\left(A_{J}, \alpha_{J}\right)_{1, P} \\
\left(B_{J}, \beta_{J}\right)_{1, Q}
\end{array}\right] V_{n}^{h_{m}, d, g_{j}}\left[p, \tau, k, w, q, k_{m}, a_{j}, b_{r}, \alpha, \beta, \delta ; y x^{\eta}\right] d x\right. \\
& =\lambda \sum_{n=0}^{\infty} \frac{(-p)^{n} \prod_{m=1}^{t}\left[\left(h_{m}\right)_{n+k_{m}}\right](d+\alpha n+\beta)^{-\tau}(y / 2)^{n k+d w+q}}{\prod_{j=1}^{S}\left[\left(g_{j}\right)_{n+a_{j}}\right] \prod_{r=1}^{u}\left[(d)_{\alpha n \delta+b_{r}}\right] c^{\mu\{\zeta+\eta(n k+d w+q)+1\} / \rho}} \\
& \quad \times H_{P+1, Q}^{M, N+1}\left[z c^{-\mu \sigma / \rho} \mid \begin{array}{l}
\left.\{-\zeta-\eta(n k+d w+q), \sigma\},\left(A_{J}, \alpha_{J}\right)_{1, P}\right] \\
\left(B_{J}, \beta_{J}\right)_{1, Q}
\end{array}\right.
\end{aligned}
$$

where

$$
A=\sum_{J=1}^{N} \alpha_{J}-\sum_{J=N+1}^{P} \alpha_{J}+\sum_{J=1}^{M} \beta_{J}-\sum_{J=M+1}^{Q} \beta_{J}
$$

and

$$
\delta=\sum_{J=1}^{Q} \beta_{J}-\sum_{J=1}^{P} \alpha_{J}
$$

provided that the conditions mentioned with (1.10) are satisfied.

Proof. We first express the general class of functions occurring in the left hand side of (3.4) in series form with the help of (1.10) and Fox's $H$-function in terms of Mellin-Barnes type contour integral wth the help of (1.8). Now, changing the order of integrations and summation and also inverting the order of contour and $x$ integrals, which is permissible under the conditions mentioned with the theorem, we get left hand side (say $\Delta$ ) as follows:

$$
\begin{aligned}
\Delta & =\lambda \sum_{n=0}^{\infty} \frac{(-p)^{n} \prod_{m=1}^{t}\left[\left(h_{m}\right)_{n+k_{m}}\right](d+\alpha n+\beta)^{-\tau}(y / 2)^{n k+d w+q}}{\prod_{j=1}^{s}\left[\left(g_{j}\right)_{n+a_{j}}\right] \prod_{r=1}^{u}\left[(d)_{\alpha n \delta+b_{r}}\right]} \\
& \times \int_{L} \phi(\xi) z^{\xi}\left[\int_{0}^{\infty} e^{-c^{\mu / \rho} x} x^{\zeta+\eta(n k+d w+q)+\sigma \xi} d x\right] d \xi .
\end{aligned}
$$

Now we substitute $c^{\mu / \rho} x=\omega$ and we get left hand side (say $\Delta$ ) as follows:

$$
\begin{aligned}
\Delta & =\lambda \sum_{n=0}^{\infty} \frac{(-p)^{n} \prod_{m=1}^{t}\left[\left(h_{m}\right)_{n+k_{m}}\right](d+\alpha n+\beta)^{-\tau}(y / 2)^{n k+d w+q}}{\prod_{j=1}^{s}\left[\left(g_{j}\right)_{n+a_{j}}\right] \prod_{r=1}^{u}\left[(d)_{\alpha n \delta+b_{r}}\right]} \\
& \times \int_{L} \phi(\xi) z^{\xi} \frac{1}{c^{\mu\{\zeta+\eta(n k+d w+q)+\sigma \xi+1\} / \rho}}\left[\int_{0}^{\infty} e^{-\omega} \omega^{\zeta+\eta(n k+d w+q)+\sigma \xi} d \omega\right] d \xi,
\end{aligned}
$$


where $\mu, \rho, \eta, \sigma$ are positive real numbers; $\operatorname{Re}(c)>0, A>0,|\arg z|<\frac{1}{2} A \pi, \delta>0 ; \operatorname{Re}\{\zeta+$ $\left.\eta(n k+d w+q)+\sigma \min _{1 \leq J \leq M}\left(\frac{B_{J}}{\beta_{J}}\right)\right\}>-1$ and the conditions mentioned with (1.10) are satisfied. $A$ and $\delta$ are given by (3.5) and (3.6) respectively.

Now, using the result (3.3) in (3.8) and interpreting the resulting contour integral in terms of $H$-function, we arrive at the right hand side of (3.4).

\section{Special cases}

In this section, we mention some special cases of the results (3.1) and (3.4) as the $H$-function and general class of functions are reducible to a large number of special functions due to their general nature.

(i) If we take $\eta=1, p=1, m=1, j=2, r=1, h_{1}=1, g_{1}=1, g_{2}=1, \tau=1, k=2, w=$ $1, q=0, k_{1}=0, a_{1}=0, a_{2}=0, b_{1}=0, \alpha=1, \beta=0, \delta=1$ and $\lambda=1 / \Gamma(d)$ in (3.1), the general class of functions reduces to the Bessel function [4] and we get the following result:

$$
L^{*}\left\{x^{d} J_{d}(y x) ; c\right\}=(2 y)^{d} \pi^{-1 / 2} \Gamma\left(d+\frac{1}{2}\right)\left(c^{2 \mu / \rho}+y^{2}\right)^{\frac{-2 d-1}{2}},
$$

where $J_{d}(z)$ stands for the Bessel function of the first kind; $\mu$ and $\rho$ are positive real numbers; $\operatorname{Re}(d)>-1 / 2$ and $c^{\mu / \rho}>|\operatorname{Im} y|$.

(ii) If we take $\mu=1$ and $\rho=1$ in (4.1), we get the following known result [6, p. 182, Eq.(7)]:

$$
L\left\{x^{d} J_{d}(y x) ; c\right\}=(2 y)^{d} \pi^{-1 / 2} \Gamma\left(d+\frac{1}{2}\right)\left(c^{2}+y^{2}\right)^{\frac{-2 d-1}{2}},
$$

provided that $\operatorname{Re}(d)>-1 / 2$ and $\operatorname{Re}(c)>|\operatorname{Im} y|$.

(iii) If we take $n=0, d=1, m=1, j=1, r=1, \tau=1, w=0, q=0, k_{1}=0, a_{1}=0, b_{1}=$ $0, \beta=0, \delta=0$ and $\lambda=1$ in (3.4), the general class of functions reduces to unity and we get the following result:

$$
L^{*}\left\{x^{\zeta} H_{P, Q}^{M, N}\left[z x^{\sigma} \mid \begin{array}{l}
\left(A_{J}, \alpha_{J}\right)_{1, P} \\
\left(B_{J}, \beta_{J}\right)_{1, Q}
\end{array}\right] ; c\right\}=c^{-\mu(\zeta+1) / \rho} H_{P+1, Q}^{M, N+1}\left[z c^{-\mu \sigma / \rho} \mid \begin{array}{l}
(-\zeta, \sigma),\left(A_{J}, \alpha_{J}\right)_{1, P} \\
\left(B_{J}, \beta_{J}\right)_{1, Q}
\end{array}\right],
$$

provided that $\mu, \rho, \sigma$ are positive real numbers; $\operatorname{Re}(c)>0, A>0,|\arg z|<\frac{1}{2} A \pi, \delta>0$ and $\operatorname{Re}\left\{\zeta+\sigma \min _{1 \leq J \leq M}\left(\frac{B_{J}}{\beta_{J}}\right)\right\}>-1$. $A$ and $\delta$ are given by (3.5) and (3.6) respectively.

(iv) If we take $\mu=1$ and $\rho=1$ in (4.3), we get the following known result [31, p. 15-16, Eq.(2.4.2)]:

$$
L\left\{x^{\zeta} H_{P, Q}^{M, N}\left[z x^{\sigma} \mid \begin{array}{l}
\left(A_{J}, \alpha_{J}\right)_{1, P} \\
\left(B_{J}, \beta_{J}\right)_{1, Q}
\end{array}\right] ; c\right\}=c^{-(\zeta+1)} H_{P+1, Q}^{M, N+1}\left[z c^{-\sigma} \mid \begin{array}{l}
(-\zeta, \sigma),\left(A_{J}, \alpha_{J}\right)_{1, P} \\
\left(B_{J}, \beta_{J}\right)_{1, Q}
\end{array}\right],
$$

provided that $\operatorname{Re}(c)>0, \sigma>0, A>0,|\arg z|<\frac{1}{2} A \pi, \delta>0$ and $\operatorname{Re}\left\{\zeta+\sigma \min _{1 \leq J \leq M}\left(\frac{B_{J}}{\beta_{J}}\right)\right\}>$ -1 . $A$ and $\delta$ are given by (3.5) and (3.6) respectively. 
(v) If we take $p=1, m=1, j=2, r=1, h_{1}=1, g_{1}=3 / 2, g_{2}=1, \tau=1, k=2, w=$ $1, q=1, k_{1}=0, a_{1}=0, a_{2}=0, b_{1}=1 / 2, \alpha=1, \beta=1 / 2, \delta=1$ and $\lambda=1 /\{\Gamma(d) \Gamma(3 / 2)\}$ in (3.1) and (3.4), the general class of functions reduces to the Struve's function [4] and we get the following results respectively:

$$
L^{*}\left\{x^{d} H_{d}\left(y x^{\eta}\right)\right\}=\sum_{n=0}^{\infty} \frac{(-1)^{n}(y / 2)^{2 n+d+1} \Gamma\{d+\eta(2 n+d+1)+1\}}{\Gamma(3 / 2+n) \Gamma(d+3 / 2+n) c^{\mu\{d+\eta(2 n+d+1)+1\} / \rho}},
$$

provided that $\mu$ and $\rho$ are positive real numbers and $\operatorname{Re}\{d+\eta(2 n+d+1)\}>-1$.

$$
\begin{aligned}
& L^{*}\left\{x^{\zeta} H_{P, Q}^{M, N}\left[z x^{\sigma}\left[\begin{array}{l}
\left(A_{J}, \alpha_{J}\right)_{1, p} \\
\left(B_{J}, \beta_{J}\right) 1, Q
\end{array}\right] H_{d}\left(y x^{\eta}\right) ; c\right\}=\sum_{n=0}^{\infty} \frac{(-1)^{n}(y / 2)^{2 n+d+1} c^{-\mu\{\zeta+\eta(2 n+d+1)+1\} / \rho}}{\Gamma(3 / 2+n) \Gamma(d+3 / 2+n)}\right. \\
& \times H_{P+1, Q}^{M, N+1}\left[z c^{-\mu \sigma / \rho} \mid \begin{array}{l}
\{-\zeta-\eta(2 n+d+1), \sigma\},\left(A_{J}, \alpha_{J}\right)_{1, P} \\
\left(B_{J}, \beta_{J}\right)_{1, Q}
\end{array},\right.
\end{aligned}
$$

provided that $\mu, \rho, \eta, \sigma$ are positive real numbers; $\operatorname{Re}(c)>0, A>0,|\arg z|<\frac{1}{2} A \pi, \delta>0$ and $\operatorname{Re}\left\{\zeta+\eta(2 n+d+1)+\sigma \min _{1 \leq J \leq M}\left(\frac{B_{J}}{\beta_{J}}\right)\right\}>-1 . A$ and $\delta$ are given by (3.5) and (3.6) respectively, and $H_{d}(z)$ stands for the Struve's function.

(vi) If we take $\eta=1, d=1 / 2, \mu=1$ and $\rho=1$ in (4.5), we get the following known result [6, p. 206, Eq.(10)]:

$$
L\left\{x^{1 / 2} H_{1 / 2}(z x)\right\}=(2)^{1 / 2} \pi^{-1 / 2} z^{3 / 2} c^{-1}\left(c^{2}+z^{2}\right)^{-1},
$$

provided that $\operatorname{Re}(c)>|\operatorname{Im} z|$.

(vii) If we take $\eta=1, p=1, m=1, j=2, r=1, h_{1}=1, g_{1}=\left(u^{\prime}+v^{\prime}+3\right) / 2, g_{2}=$ $\left(u^{\prime}-v^{\prime}+3\right) / 2, d=1, \tau=1, k=2, w=u^{\prime}, q=1, k_{1}=0, a_{1}=0, a_{2}=0, b_{1}=-1, \alpha=$ $1, \beta=-1, \delta=1$ and $\lambda=2^{u^{\prime}+1} /\left(u^{\prime} \pm v^{\prime}+1\right)$ in (3.1) and (3.4), the general class of functions reduces to the Lommel's function [4] and we get the following results respectively:

$$
\begin{aligned}
L^{*}\left\{x^{d} s_{u^{\prime}, v^{\prime}}(y x)\right\} & =\frac{y^{u^{\prime}+1} \Gamma\left(u^{\prime}+3\right)}{\left(u^{\prime} \pm v^{\prime}+1\right) c^{\mu\left(u^{\prime}+3\right) / \rho}} \\
& \times{ }_{3} F_{2}\left[1, \frac{u^{\prime}+3}{2}, \frac{u^{\prime}+4}{2} ; \frac{u^{\prime}+v^{\prime}+3}{2}, \frac{u^{\prime}-v^{\prime}+3}{2} ; \frac{-y^{2}}{c^{2 \mu / \rho}}\right],
\end{aligned}
$$

provided that $\mu$ and $\rho$ are positive real numbers and $\operatorname{Re}\left(u^{\prime}\right)>-3$.

$$
\begin{aligned}
& L^{*}\left\{x^{\zeta} H_{P, Q}^{M, N}\left[z x^{\sigma}\left[\begin{array}{l}
\left(A_{J}, \alpha_{J}\right)_{1, P} \\
\left(B_{J}, \beta_{J}\right)_{1, Q}
\end{array}\right] s_{u^{\prime}, v^{\prime}}\left(y x^{\eta}\right) ; c\right\}\right. \\
& =\frac{2^{u^{\prime}+1}}{u^{\prime} \pm v^{\prime}+1} \sum_{n=0}^{\infty} \frac{(-1)^{n}(y / 2)^{2 n+u^{\prime}+1} c^{-\mu\left\{\zeta+\eta\left(2 n+u^{\prime}+1\right)+1\right\} / \rho}}{\left(\frac{u^{\prime} \pm v^{\prime}+3}{2}\right)_{n}} \\
& \times H_{P+1, Q}^{M, N+1}\left[z c^{-\mu \sigma / \rho} \mid \begin{array}{l}
\left\{-\zeta-\eta\left(2 n+u^{\prime}+1\right), \sigma\right\},\left(A_{J}, \alpha_{J}\right)_{1, P} \\
\left(B_{J}, \beta_{J}\right)_{1, Q}
\end{array}\right]
\end{aligned}
$$


provided that $\mu, \rho, \eta, \sigma$ are positive real numbers; $\operatorname{Re}(c)>0, A>0,|\arg z|<\frac{1}{2} A \pi, \delta>0$ and $\operatorname{Re}\left\{\zeta+\eta\left(2 n+\mu^{\prime}+1\right)+\sigma \min _{1 \leq J \leq M}\left(\frac{B_{J}}{\beta_{J}}\right)\right\}>-1 . A$ and $\delta$ are given by (3.5) and (3.6) respectively, and $s_{u^{\prime}}, v^{\prime}(z)$ stands for the Lommel's function.

(viii) If we take $p=-2, m=1, j=1, r=1, h_{1}=h, g_{1}=1, \tau=1, k=1, w=0, q=$ $0, k_{1}=0, a_{1}=0, b_{1}=-1, \beta=-1, \delta=1$ and $\lambda=1 / \Gamma(d)$ in (3.1) and (3.4), the general class of functions reduces to the generalized Mittag-Leffler function introduced and studied by Prabhakar [26] and we get the following results respectively:

$$
L^{*}\left\{x^{d} E_{\alpha, d}^{h}\left(y x^{\eta}\right) ; c\right\}=\sum_{n=0}^{\infty} \frac{(h)_{n} \Gamma(d+\eta n+1) y^{n}}{n ! \Gamma(d+\alpha n) c^{\mu(d+\eta n+1) / \rho}},
$$

provided that $\mu$ and $\rho$ are positive real numbers and $\operatorname{Re}(d+\eta n)>-1$.

$$
\begin{aligned}
L^{*}\left\{x^{\zeta} H_{P, Q}^{M, N}\left[z x^{\sigma} \mid \begin{array}{c}
\left(A_{J}, \alpha_{J}\right)_{1, P} \\
\left(B_{J}, \beta_{J}\right)_{1, Q}
\end{array}\right] E_{\alpha, d}^{h}\left(y x^{\eta}\right) ; c\right\} & =\sum_{n=0}^{\infty} \frac{(h)_{n} y^{n} c^{-\mu(\zeta+\eta n+1) / \rho}}{\Gamma(d+\alpha n) n !} \\
& \times H_{P+1, Q}^{M, N+1}\left[z c^{-\mu \sigma / \rho} \mid \begin{array}{l}
(-\zeta-\eta n, \sigma),\left(A_{J}, \alpha_{J}\right)_{1, P} \\
\left(B_{J}, \beta_{J}\right)_{1, Q}
\end{array}\right],
\end{aligned}
$$

provided that $\mu, \rho, \eta, \sigma$ are positive real numbers; $\operatorname{Re}(c)>0, A>0,|\arg z|<\frac{1}{2} A \pi, \delta>0$ and $\operatorname{Re}\left\{\zeta+\eta n+\sigma \min _{1 \leq J \leq M}\left(\frac{B_{J}}{\beta_{J}}\right)\right\}>-1 . A$ and $\delta$ are given by (3.5) and (3.6) respectively, and $E_{\alpha, d}^{h}(z)$ stands for the generalized Mittag-Leffler function.

If we substitute $h=1$ in (4.10) and (4.11), the generalized Mittag-Leffler function reduces to the generalized Mittag-function $E_{\alpha, d}\left(y x^{\eta}\right)$ introduced and studied by Wiman [34].

If we substitute $h=1, d=1$ in (4.10) and (4.11), the generalized Mittag-Leffler function reduces to the Mittag-Leffler function $E_{\alpha}\left(y x^{\eta}\right)[11,12]$.

(ix) If we take $p=-2, m=1, j=1, r=1, h_{1}=h, g_{1}=1, k=1, w=0, q=0, k_{1}=$ $0, a_{1}=0, b_{1}=0, \alpha=1, \beta=0, \delta=0$ and $\lambda=1$ in (3.1) and (3.4), the general class of functions reduces to the unified Riemann-zeta function [10] and we get the following results respectively:

$$
L^{*}\left\{x^{d} \phi_{h}\left(y x^{\eta}, \tau, d\right) ; c\right\}=\sum_{n=0}^{\infty} \frac{(h)_{n} \Gamma(d+\eta n+1) y^{n}}{n !(d+n)^{\tau} c^{\mu(d+\eta n+1) / \rho}}
$$

provided that $\mu$ and $\rho$ are positive real numbers and $\operatorname{Re}(d+\eta n)>-1$.

$$
\begin{aligned}
& L^{*}\left\{x^{\zeta} H_{P, Q}^{M, N}\left[z x^{\sigma} \mid \begin{array}{l}
\left(A_{J}, \alpha_{J}\right)_{1, P} \\
\left(B_{J}, \beta_{J}\right)_{1, Q}
\end{array}\right] \phi_{h}\left(y x^{\eta}, \tau, d\right) ; c\right\} \\
& =\sum_{n=0}^{\infty} \frac{(h)_{n} y^{n} c^{-\mu(\zeta+\eta n+1) / \rho}}{(d+n)^{\tau} n !} H_{P+1, Q}^{M, N+1}\left[z c^{-\mu \sigma / \rho} \mid \begin{array}{l}
\left.(-\zeta-\eta n, \sigma),\left(A_{J}, \alpha_{J}\right)_{1, P}\right], \\
\left(B_{J}, \beta_{J}\right)_{1, Q}
\end{array}\right]
\end{aligned}
$$

provided that $\mu, \rho, \eta, \sigma$ are positive real numbers; $\operatorname{Re}(c)>0, A>0,|\arg z|<\frac{1}{2} A \pi, \delta>0$ and $\operatorname{Re}\left\{\zeta+\eta n+\sigma \min _{1 \leq J \leq M}\left(\frac{B_{J}}{\beta_{J}}\right)\right\}>-1 . A$ and $\delta$ are given by (3.5) and (3.6) respectively, and $\phi_{h}(z, \tau, d)$ stands for the unified Riemann-zeta function. 
If we substitute $h=1$ in (4.12) and (4.13), the unified Riemann-zeta function reduces to the Hurwitz-Lerch zeta function [3, p. 27, Eq. (1)] which reduces to the generalized zeta function [3, p. 24, Eq. (1)] when we substitute $y=1, x=1$ and Riemann-zeta function [3, p. 32, Eq. (1)] when $y=1, x=1$ and $d=1$.

$(x)$ If we take $p=2, r=1, d=1, t=P^{\prime}, s=Q^{\prime}, \tau=1, k=1, w=0, q=0, k_{m}=0, a_{j}=$ $0, b_{1}=-1, \alpha=1, \beta=-1, \delta=1$ and $\lambda=\frac{\prod_{m=1}^{P^{\prime}} \Gamma\left(h_{m}\right)}{\prod_{j=1}^{Q^{\prime}} \Gamma\left(g_{j}\right)}$ in (3.4), the general class of functions reduces to the MacRobert's E-functioin [3] and we get the following result:

$$
\begin{aligned}
& L^{*}\left\{x^{\zeta} H_{P, Q}^{M, N}\left[z x^{\sigma} \mid \begin{array}{l}
\left(A_{J}, \alpha_{J}\right)_{1, P} \\
\left(B_{J}, \beta_{J}\right)_{1}, Q
\end{array}\right] E\left[P^{\prime} ;\left(h_{P^{\prime}}\right) ; Q^{\prime} ;\left(g_{Q^{\prime}}\right) ; \frac{1}{y x^{\eta}}\right] ; c\right\} \\
& =\frac{\prod_{m=1}^{P^{\prime}} \Gamma\left(h_{m}\right)}{\prod^{\prime} \Gamma\left(g_{j}\right)} \sum_{n=0}^{\infty} \frac{\prod_{m=1}^{P^{\prime}}\left(h_{m}\right)_{n}(-y)^{n} c^{-\mu(\zeta+\eta n+1) / \rho}}{\prod_{j=1}^{Q^{\prime}}\left(g_{j}\right)_{n} n !} \\
& \times H_{P+1, Q}^{M, N+1}\left[z c^{-\mu \sigma / \rho} \mid \begin{array}{l}
\left.(-\zeta-\eta n, \sigma),\left(A_{J}, \alpha_{J}\right)_{1, P}\right], \\
\left(B_{J}, \beta_{J}\right)_{1, Q},
\end{array}\right.
\end{aligned}
$$

provided that $\mu, \rho, \eta, \sigma$ are positive real numbers; $\operatorname{Re}(c)>0, A>0,|\arg z|<\frac{1}{2} A \pi, \delta>0$ and $\operatorname{Re}\left\{\zeta+\eta n+\sigma \min _{1 \leq J \leq M}\left(\frac{B_{J}}{\beta_{J}}\right)\right\}>-1$. A and $\delta$ are given by (3.5) and (3.6) respectively, and $E\left[P^{\prime} ;\left(h_{P^{\prime}}\right) ; Q^{\prime} ;\left(g_{Q^{\prime}}\right) ; \frac{1}{z}\right]$ stands for the MacRobert's E-function.

(xi) If we take $p=2, m=1, j=2, r=1, h_{1}=1, g_{1}=1, g_{2}=1, \tau=1, k=1, w=$ $0, q=0, k_{1}=0, a_{1}=0, a_{2}=0, b_{1}=0, \beta=0, \delta=1$ and $\lambda=\frac{1}{\Gamma(d)}$ in (3.4), the general class of functions reduces to the Wright's generalized Bessel function [35] and we get the following result:

$$
\begin{aligned}
& L^{*}\left\{x^{\zeta} H_{P, Q}^{M, N}\left[z x^{\sigma} \mid \begin{array}{l}
\left(A_{J}, \alpha_{J}\right)_{1, P} \\
\left(B_{J}, \beta_{J}\right)_{1, Q}
\end{array}\right] J_{d}^{\alpha}\left(y x^{\eta}\right) ; c\right\} \\
& =\sum_{n=0}^{\infty} \frac{(-y)^{n} c^{-\mu(\zeta+\eta n+1) / \rho}}{\Gamma(d+\alpha n+1) n !} H_{P+1, Q}^{M, N+1}\left[z c^{-\mu \sigma / \rho} \mid \begin{array}{l}
(-\zeta-\eta n, \sigma),\left(A_{J}, \alpha_{J}\right)_{1, P} \\
\left(B_{J}, \beta_{J}\right)_{1, Q}
\end{array}\right],
\end{aligned}
$$

provided that $\mu, \rho, \eta, \sigma$ are positive real numbers; $\operatorname{Re}(c)>0, A>0,|\arg z|<\frac{1}{2} A \pi, \delta>0$ and $\operatorname{Re}\left\{\zeta+\eta n+\sigma \min _{1 \leq J \leq M}\left(\frac{B_{J}}{\beta_{J}}\right)\right\}>-1$. A and $\delta$ are given by (3.5) and (3.6) respectively, and $J_{d}^{\alpha}(z)$ stands for the Wright's generalized Bessel function. 
(xii) If in (4.11), we set $M=N=P=Q-1=1, h=1$ and use the identity [5, Section 18.1]

$$
E_{\beta, \delta}(z)=H_{1,2}^{1,1}\left[-z \mid \begin{array}{l}
(0,1) \\
(0,1),(1-\delta, \beta)
\end{array}\right]
$$

where $E_{\beta, \delta}(z)$ stands for the Mittag-Leffler function defined in the monograph by Erdélyi et al. [4] as

$$
E_{\beta, \delta}(z)=\sum_{k=0}^{\infty} \frac{z^{k}}{\Gamma(\beta k+\delta)}, \operatorname{Re}(\beta)>0, \operatorname{Re}(\delta)>0,
$$

the $H$-function reduces to the Mittag-leffler function and consequently we get the following interesting result associated with a product of two Mittag-leffler functions:

$$
\begin{aligned}
L^{*}\left\{x^{\zeta} E_{\beta, \delta}\left(-z x^{\sigma}\right) E_{\alpha, d}\left(y x^{\eta}\right) ; c\right\} & =\sum_{n=0}^{\infty} \frac{y^{n} c^{-\mu(\zeta+\eta n+1) / \rho}}{\Gamma(d+\alpha n)} \\
& \times H_{2,2}^{1,2}\left[\begin{array}{ll}
\left.z c^{-\mu \sigma / \rho} \mid \begin{array}{l}
(-\zeta-\eta n, \sigma),(0,1) \\
(0,1),(1-\delta, \beta)
\end{array}\right] .
\end{array}\right.
\end{aligned}
$$

\section{Application}

In this section we apply the integral transform defined by (1.5) to solve a differential equation.

Lemma 5.1. Let $f(x)$ be continuous function for all $x \geq 0$ and be of exponential orders as $x \rightarrow \infty$ and if $f^{\prime}(x)$ is of class $A$, then the Laplace transform of $f^{\prime}(x)$ exists when $s>a$ and is given by

$$
L\left\{f^{\prime}(x)\right\}=s L\{f(x)\}-f(0) .
$$

Lemma 5.2. Substituting $s=c^{\mu / \rho}$ in (5.1) and using the result (1.7) we get the following result for the generalized fractional Laplace transform:

$$
L^{*}\left\{f^{\prime}(x)\right\}=c^{\mu / \rho} L^{*}\{f(x)\}-f(0) .
$$

Lemma 5.3. Let $f(x)$ and its derivatives $f^{\prime}(x), f^{\prime \prime}(x), \ldots, f^{n-1}(x)$ be continuous functions for all $x \geq 0$ and be of exponential orders as $x \rightarrow \infty$ and if $f^{n}(x)$ is of class $A$, then the generalized fractional Laplace transform of $f^{n}(x)$ is given by

$$
L^{*}\left\{f^{n}(x)\right\}=c^{n \mu / \rho} L^{*}\{f(x)\}-\sum_{r=1}^{n} c^{\mu(n-r) / \rho} f^{r-1}(0) .
$$

Proof. Using the result (5.2), we can easily prove (5.3).

Lemma 5.4. Using integration by parts we can easily prove the following result:

$$
L^{*}(\cos a x)=\frac{c^{\mu / \rho}}{c^{2 \mu / \rho}+a^{2}}, c>0 .
$$

Example 5.1. To solve the differential equation

$$
\left(D^{2}+1\right) y=0, y(0)=1, y^{\prime}(0)=0,
$$


we take the generalized fractional Laplace transform of both sides of the differential equation and we get

$$
L^{*}\left(y^{\prime \prime}\right)+L^{*}(y)=0,
$$

or

$$
c^{2 \mu / \rho} L^{*}(y)-c^{\mu / \rho} y(0)-y^{\prime}(0)+L^{*}(y)=0,
$$

or

$$
c^{2 \mu / \rho} L^{*}(y)-c^{\mu / \rho} .1-0+L^{*}(y)=0,
$$

or

$$
L^{*}(y)=\frac{c^{\mu / \rho}}{c^{2 \mu / \rho}+1}=L^{*}(\cos x),
$$

or

$$
y=\cos x
$$

which is the required solution of the differential equation (5.5).

\section{Concluding remark}

In our present investigation, we have obtained the images of some special functions in the generalized fractional Laplace transform. Substituting $\mu=0$ and $\rho=0$ in our results, we can obtain the images of concerned special functions in the conventional Laplace transform.

\section{Acknowledgments}

The author is extremely thankful to the worthy referees for the valuable suggestions given for the improvement of the present paper.

\section{References}

[1] B. R. Bhonsle, A relation between Laplace and Hankel transforms, Proc. Glasgow Math. Assoc., 5, No. 3 (1962), 114-115.

[2] B. R. Bhonsle, A relation between Laplace and Hankel transforms, Math. Japon., 10 (1965), 85-89.

[3] A. Erdélyi, W. Magnus, F. Oberhettinger and F. G. Tricomi, Higher Transcendental Functions, Vol I, McGrawHill Book Company, New York, Toronto and London (1953).

[4] A. Erdélyi, W. Magnus, F. Oberhettinger and F. G. Tricomi, Higher Transcendental Functions, Vol II, McGrawHill Book Company, New york, Toronto and London (1953).

[5] A. Erdélyi, W. Magnus, F. Oberhettinger and F. G. Tricomi, Higher Transcendental Functions, Vol III, McGrawHill Book Company, New york, Toronto and London (1953).

[6] A. Erdélyi, W. Magnus, F. Oberhettinger and F. G. Tricomi, Tables of Integral Transforms, Vol I, McGraw-Hill Book Company, New York (1954).

[7] K. C. Gupta and S. M. Agrawal, Unified theorems involving $H$-function transform and Meijer Bessel function transform, Proc. Indian Acad. Sci., 96, No. 2 (1987), 125-130.

[8] S. P. Goyal and S. K. Vasishta, Certain relations between generalized Kontorovitch-Lebdev transform and $H$-function transform, Ranchi Univ. Math. Jour., 6 (1975), 95-102. 
[9] S. P. Goyal and R. M. Jain, Certain results for two-dimensional Laplace transform with applications, Proc. Nat. Acad. Sci. India, 59(A), No. (III) (1989), 407-414.

[10] S. P. Goyal and R. K. Laddha, On the generalized Riemann zeta functions and the generalized Lambert transform, Ganita Sandesh, 11, No. 2 (1997), 99-108.

[11] P. Humbert and R. P. Agarwal, Sur la fonction de Mittag-Leffler et quelques unes de ses generalizations , Bull. Sci. Math., 77, No. 2 (1953), 180-185.

[12] V. Kumar, A general class of functions and N-fractional calculus, J. Rajasthan Acad. Phys. Sci., 11, No. 3 (2012), 223-230.

[13] V. Kumar, $N$-fractional calculus of general class of functions and Fox's $H$-function, Proc. Natl. Acad. Sci., sect. A, Phys. Sci. 83, No. 3 (2013), 271-277.

[14] V. Kumar, The Euler transform of $V$ - function, Afr. Mat., 29, No. (1-2) (2018), 23-27

[15] V. Kumar, Theorems connecting Mellin and Hankel transforms, J. Classical Anal., 15, No. 2 (2019), 123-130.

[16] V. Kumar, Theorems connecting Fourier sine transform and Hankel transform, SeMA Journal, 77, No. 2 (2020), 219-225.

[17] V. Kumar, Theorems connecting Stieltjes transform and Hankel transform, São Paulo J. Math. Sci., DOI: $10.1007 /$ s40863-020-00182-4.

[18] V. Kumar, On a General Theorem Connecting Laplace Transform and Generalized Weyl fractional Integral Operator Involving Foxs H-Function and a General Class of Functions, J. Frac. Calc. and Appl., 11, No. 2 (2020), 270-280.

[19] V. Kumar, On a Generalized Fractional Fourier Transform, Palestine J. Maths., 9, No. 2 (2020), 903-907.

[20] A. A. Kilbas and M. Saigo, H-Transforms: Theory and Application, Chapman \& Hall/CRC Press, Boca Raton, London, New york (2004).

[21] G. M. Mittag-Leffler, Sur la nouvelle function $E_{\alpha}(x)$, C. R. Acad. Sci. Paris, 137 (1903), 554-558.

[22] A. M. Mathai, R. K. Saxena and H. J. Haubold, The H-Function: Theory and Applications. Springer, New york (2010).

[23] G. D, Medina, N. R. Ojeda, J. H. Pereira and L. G. Romero, Fractional Laplace transform and fractional calculus, Inter. Math. Forum, 12, No. 20 (2017), 991-1000.

[24] A. P. Prudnikov, Yu. A. Brychkov and O. I. Marichev, Integrals and Series, vol. 3, More Special Functions, Gordon and Breach Science Publishers, New york (1990).

[25] T. R. Prabhakar, A singular integral equation with a generalized Mittag-Leffler function in the kernel, Yokohama Math. J., 19 (1971), 7-15.

[26] L. G. Romero, G. D. Medina, N. R. Ojeda and J. H. Pereira, A new alfa-integral Laplace transform, Asian J. Current Engg. Maths., 5 (2016), 59-62.

[27] H. M. Srivastava, A relation between Meijer and Hankel transforms, Math. Japon., 11 (1966), 11-13.

[28] H. M. Srivastava, On a relation between Laplace and Hankel transforms, Matematiche (Catania), 21 (1966), 199-202.

[29] H. M. Srivastava, Some remarks on a generalization of the Stieltjes transform, Publ. Math. Debrecen, 23 (1976), 119-122.

[30] H. M. Srivastava, K. C. Gupta and S. P. Goyal, The H-function of one and two Variables with Applications, South Asian Publishers, New Delhi (1982).

[31] H. M. Srivastava and V. K. Tuan, A new convolution theorem for the Stieltjes transform and its application to a class of singular integral equations, Arch. Math.(Basel), 64, No. 2 (1995), 144-149.

[32] H. M. Srivastava and O. Yürekli, A theorem on a Stieltjes-type integral transform and its applications to obtain infinite integrals of elementary and special functions, Complex Vari., Theory Appl., 28, No. 2 (1995), 159-168.

[33] A. Wiman, Über den Fundamentalsatz in der Teorie der Funktionen $E_{\alpha}(x)$, Acta. Math., 29 (1905), 191-201.

[34] E. M. Wright, The asymptotic expansion of the generalized Bessel function, Proc. London Math. Soc., 38, No. 2 (1935), 257-270.

[35] S. Yakubovich and M. Martins, On the iterated Stieltjes transform and its convolution with applications to singular integral equations, Trans. Spec. Func., 25, No. (2013), DOI: 10.1080/10652469.2013.868457. 